\title{
Sulla propagazione magnetoguidata dei whistlers
}

\author{
(On the magnetogniding of whistlers)
}

\author{
P. THOMINic1
}

Ricevulo il 4 Magrgio 197 ]

\begin{abstract}
Rrassunto. - Viene esuminata la teoria della propagazione ilei whistlers nel tentativn di indicare le linee di una trattazione unitaria che inserisea nella teoria di Storey acquisizioni successive. Le linee di sviluppo di talo irattazione sono mostrate a partire dalle oquazioni base tella teoria magntetoionica, scritte, nell'approssimazione quasi longitultitale, in una forma più completa di quella usuale. 1 risultati principali, qualitativamente discussi, sono l'esiatenza di una corronte longitudinale nol plasma ionosforico, formalnente rapace di dar conto di alenni risultati della teoria delle colonne cli innizzazione, e l'esistenza di altre componenti magneloioniche, la strnordinaria delle quali dà conto dell'intrappolamento dei whistlers nel cono di Storey nella bassissima ionosfera, in presenza di un forte effetto rifrattivn delle collisioni elettroniche.
\end{abstract}

SUmmary. - The theory of the whistler propugation is examined in order to outline a coherent and comprehensive exposition of varions results. The line of such an expnsition are shown, starting from a more complete form of the basic magnetoionic equations in the Q.L. approximation. The principal results are qualitatively ilisenssed, numely tho offoet of a longitudinal current, equivalent to the effect of a longitudinal column of ionisation, and the excitation of a kelf-trapping extraordinary component in the lower ionosphero.

\section{1. - P'REMESSA.}

Con la locuzione " radiopropagazione magnetoguidata " intendiamo riferirei al fenomeno in virtì del quale radioonde si propagano nell'atmosfera terrestre seguendo linee di forza del campo magnetico 
terrestre, per modo che raliosegnali irralinti in un eerto pruto della Terra sono riceruti nel prunto magnetichmente coniugato. P'er quanto non manchino osservazioni di feromeni del genere anche nel campo dello radioonde di rolativamente alta fregnenza, il caso più vistoso, a anche più interessante, sli rałliopropagazione magnetogniłlata è quello dei cosiddetti whistlers: si tratta delle eomponenti di bassa frequenza (sino a cirea $10 \mathrm{kH} z$ ) tell'irraggiamento e.m. associato a scariche elottriche atmosferiche, procelenti tlispersivamente lungo le lines tli forza del c.m.t., seconto un'ipotesi, oggi nniversalmente arcettata, emessa nel 1953 da. L. R. O. Storey (17). Precisamente, sccomulo la teoria rli Storey, il "moko whistler " di propagazione $\dot{e}$, nel linguaggio lella teoria magnetoionica che preciseremo tra poco, la puopragazione longitudinale (ciò parallela al c.m.t.) clelle componenti struorelinarie regli atmosferici (cios dalle perturbazioni e.m. a largo spettro originantisi tha seariche clettriche atmosferiche).

Esula rallo scopo del presente lavoro fare una esauriente rassema clei risultati a tatt oggi acquisiti sui fonomeni di radiopropragazione magnetognislata e sui whistlers in particolare; ci limiteremo a ricorslare aleuni fatti: (a) la propagazione slei whistlers avviene con grande efficienza, essendo un fatto frequente la ricezione di treni ri whistlers regolarmente spaziati, corrisprontenti a energia e.m. che segue più volte una linea di forza tlel c.m.t., riflettenctosi alle estremità, sulla superficie terrestre, con attennazione pinttosto piccola tra un segnale e il successivo; (b) la valirlità dell ipotesi clıe è alla base della teoria rli Storey è stata verificata con whistlers artificiali tra località magneticumente coningate (3).

Ia trattazione corrente per la propagazione magnetognirlata dei whistlers, clıe per comodità seguiteremo a cliamare teorin di stovey anche se ad essa lamno contribuito varí zicercatori nel periodo successivo al 1953, fa riferimento alla teoria generale della radiopropagazione nella ionosfera, la cosidateta teoria magnetoioniea, e a particolari ipotesi sulla struttura tlell'alta ionosfera.

\section{2. - ThORI MAGNETOIONICA.}

Cominceremo con il ricordare sinteticamente la teoria magnetoioniea, dato che alcuni punti di essa risultano essenziali per la discussione che segne. La trattazione $\dot{e}$, nelle grandi linee, qnella corrente attualmente, sostanzialmente rifacentesi alla trattazione di F. F. 
Appleton (1927) ("); il simbolismo i quello raccomandato dalla IT.R.S.T. e le grandezze s'intenilono misurate in unità clel sistema SI.

Jue ipotesi esplicite a base della teoria sono le seguenti:

(I) la ionosfera ì assimilata a un magnetoplasma non caldo:

(II) li permittivitì, $\varepsilon_{0}$, e la permeabilità, $\mu_{0}$, della ionosfera sono quelle ilel ruoto;

(III) l'interazione della ionosfera con un'onda e.m. avviene soltanto per mezzo slegli clettroni liberi nella ionosfera medesima;

(I 5 ) le collisioni tra elettroni liberi eccitati dall'onda e circostanti paticelle vengono rappresentate mediante una forza di tipo viscoso, assumendo che in ogni collisione un elettrone ceda alla particella urtata l'impulso aequistato ilall'onda.

A proposito dell'ipotesi (II), va osservato che nella trattazione usuale la permenbilitì $\mu$ e la conduttiviti $\sigma$ della ionosfera sono assunte pari a quelle del vuoto $\left(\mu--\mu_{0}, \sigma=0\right)$; l'efietto del moto degli elettroni eccitati dall'onda viene valutato in termini di variazione della permittività (tensore $\equiv \neq \varepsilon_{v}$ ). Qui, invece, tale effetto ì computato in termini di variazione della conduttività (tensore $\lrcorner \neq 0$ ) 0 , che è lo stesso, della densità della corrente di conduzione: ciò ci sembra utile non solo perché $\dot{\partial}$, tutto sommato, pì̀ alerente alla realtì fisica, ma anche perchí, come si verlrǹ, porta a considerare velocitì melie elettroniche e non posizioni medie elettroniche (vale a dire, nell'equazione dei moti elettronici, derivate temporali jrime, e non seconde, degli spostamenti elettronici). Non v'è bisogno di dire che, a parte differenze formali nei calcoli intermedî, i clue punti di vista portano a risultati identici.

Consideriamo dunque un'onda piana armonica procedente nel verso positivo dell'asse $x$ di un riferimento cartesiano ordinario $(O$, $x, y, z)$; per le grandezze inerenti alla propagazione (intensiti del campo elettrico $\mathbf{E}$, induzione magnetica $B$, densità di corrente $\mathbf{j}$ ) valgono le relazioni della tabella seguente, essendo $i^{2}=-1$, (1) la pulsazione dell'onda, $f$ la frequenza, $e$ la velocità di propagazione delle onde e.m. nel vuoto, $n$ l'indice di rifrazione (cli fase), $v$ la velocitì di fase, $a$ il coefficiente lineico d'assorbimento.

(*) Un'estesa hibliografia sulla teoria magnetoioniea è riportata in $\left.{ }^{(}\right)$. 
T'ab. I
(1) $\mathbf{L}-L_{0} \exp i(\omega t-\boldsymbol{k} x)$,
(2) $k=\frac{\omega}{c} n=\frac{2 \pi f}{c} n$,
(3) $n=n-i z$,
(4) $n=\frac{c}{v}=\left(\frac{\varepsilon \mu}{\varepsilon_{0} \mu_{0}}\right)^{1 / 2}$,
(5) $\quad u=\frac{\omega}{c} \chi$.

Sfruttando tali relazioni, le equazioni di Maxwell,

(6)

$$
\text { a }\left\{\begin{array}{l}
\operatorname{rot} \mathbf{E}=-\frac{\partial \mathbf{B}}{\partial i}, \\
\operatorname{rot} \mathbf{B}=\mu_{0} \mathbf{j}+\frac{1}{c^{2}} \frac{\partial \mathbf{E}}{\partial t},
\end{array}\right.
$$

dimno:

i)
a. $B_{x}=0$,
b $B_{y}=-\frac{k}{(t)} W_{z}$,
c $R_{z}=\frac{k}{(1)} E_{n}$,
a. $j j_{x}=-i \varepsilon_{0}(1) H_{x}$,
$\mathrm{b} ! j_{y}=i \varepsilon_{0}(n)\left(n^{2}-1\right) E_{y}$,
c $j_{z}=i \varepsilon_{0}(1)(n=-1) E_{z}$.

Ja (7)a mostra che il campo magnetico i trasversale; il campo elettrico non ì invece, in generale, trasversale. Per caratterizzare lo stato di polarizzazione dell'onsla nel piano $y z$ introrluciamo la quantiti

$$
\boldsymbol{R}=-\frac{B_{y}}{B_{z}}-\frac{E_{z}}{E_{y}}-\frac{j_{z}}{j_{y}},
$$

mentre per caratterizzare lo stato di polarizzazione dell'onda elettrica. nel piano $x y$ introduciamo la quantitì

$$
\boldsymbol{S}=\frac{E_{x}}{E_{y}} .
$$

$\Lambda$ valori reali oppure complessi di $\boldsymbol{R}$ e $\boldsymbol{S}$ corrisponde una polarizzazione lineare oppure ellittica; in particolare, si ha polarizzazione circolare per $R$ o $S$ pari a \pm .

Supponiamo ora che il trierlro di riferimento sia scelto in modo che l'induzione $\mathbf{B}_{n}$ del c.m.t. giaccia nel piano $x y$, avento compo- 
nente $B_{T}$, secondo l'asse $x$ e componente $B_{T}$ secondo l'asse $y$ (i deponenti $L$ e $T$ stanno, qui e nel seguito, per " longitudinale " $e$ " trasversale", con riferimento alla slirezione di propagazione dellonda rispetto a quella del c.m.t.). Non portando in conto il campo magnetico dell'onda e portanklo in conto, come detto, un termine di resistenza viscosa per le tollisioni elettroniche, l'equazione del moto medio di un detirone eccitato dall'onsla i la seguente:

$$
m \ddot{\mathbf{r}}=q \mathbf{E}+q \dot{\mathbf{r}} \times \mathbf{B}_{H}-m \nu \dot{\mathbf{r}}
$$

essendo $\mathrm{r}$ il rettore inslividuante la posizione dell'elettrone, $q$ e $m$ la carien e la massa clell'elettrone, " la frequenza delle collisioni elettroniche.

Indicando con $\mathbf{r}$ la velociti media degli $A$ elettroni per unita di volume, è, a norma di definizione,

$$
\mathbf{j}=N q \overline{\mathbf{x}}
$$

ricoulando la (1) e approssimando $d / d t \cong$ ojor, si ha:

$$
\overline{\overrightarrow{\mathrm{r}}}=\frac{1}{N q} i \omega \mathbf{j} \text {. }
$$

Ja. (11) diventa così:

$$
-\mathbf{j}=i \frac{N q^{2}}{m \omega} \mathbf{E}+i \frac{q}{m \omega} \mathbf{j} \times \mathbf{B}_{H}-i \frac{p}{\omega} \mathbf{j} .
$$

Facciamo le seguenti posizioni:

J'ab. II

(15)

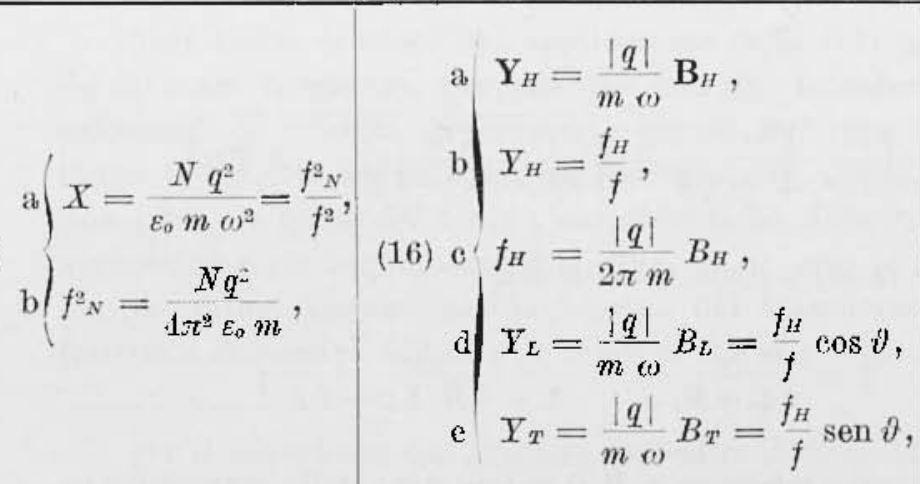

(17) $Z=\frac{v}{\omega}=\frac{v}{2 \pi f}$, 
essendo $f_{N}$ la frequenza di plasma, $f_{H}$ la frequenza di ciclotrone, o girofrequenza, of l'angolo che la direzione di propagazione forma con la direzione del c.m.t. Le grandezze $X$ e $Y$ dipendono, come si vede, dalla carica speciliea, talché risulta lucito, almeno in prima approssimazione, considerare il solo eftetto degli elettroni. Introrluendo nella (14) le grandezze ora definite e proiettando sugli assi, si hammo le tre equazioni scalari:

$$
\begin{aligned}
& \text { a }\left(-j_{x}-i \varepsilon_{0} \omega X E_{x}+i j_{z} Y_{T}-i Z j_{x},\right. \\
& \mathrm{b}-j_{y}-i \varepsilon_{0} \omega X E_{y}+i j_{z} Y_{L}-i Z j_{y}, \\
& \mathrm{c}\left(-j_{z}-i \varepsilon_{0}(1) X E_{z}+i j_{x} Y_{y}-i j_{y} Y_{L}-i Z j_{z} .\right.
\end{aligned}
$$

A norma delle (9) e (10), i $j_{z}=R j_{y}, K_{z}=R E_{y}, j_{x}=S j_{y f}\left(1-n^{2}\right)$; operando opportune sostituzioni nelle (18)b e (18)c, e quindi confrontando le nuove espressioni si ottiene:

$$
S=\frac{I_{t}}{V_{T}}\left(1+R^{2}\right)\left(1-n^{2}\right) .
$$

I)'altro canto, operando analogamente nella (18)a e tenendo conto della (8)a si ha:

$$
S=\frac{i Y_{-}}{1-\bar{I}-i \bar{L}} R\left(1-n^{2}\right)
$$

Eliminando $\boldsymbol{S}$ tra queste due relazioni si ha per $\boldsymbol{R}$ l'equazione

$$
Y_{I}, R^{2}-\frac{i Y_{g^{i}}}{1-\lambda-i Z} R+Y_{i s}=0
$$

che, risolta, dà:

$$
R=\frac{i}{2 Y_{L}}\left(\frac{X^{2} T}{1-X-i Z}=F \sqrt{\left.\frac{Y^{4}{ }^{\prime}}{(1-X-i Z)^{2}}+4 Y^{2_{L}}\right)} .\right.
$$

Ricordando la (8)b, dalla (18)b si ha:

$$
n^{2}-1=\frac{j_{y}}{i \varepsilon_{0}(1) E_{y}}=-\frac{X}{1+i R Y_{L}-i Z}
$$

sostituendo in questa relazione a $R$ il valore dato dalla (21), si ha in- 
line la formula ai Appletom (*):

$$
\begin{aligned}
& n^{2}=(n-i \chi)^{2}= \\
& =1-\frac{X}{1-i Z-\frac{Y_{T}^{2}}{2(1-X-i Z)} \pm \sqrt{\frac{Y_{T^{4}}}{4(1-X-i Z)^{2}}+Y_{L}{ }^{2}} .}
\end{aligned}
$$

Tella ionosfera si cecitano dunque due onde (componenti magnetoioniche), della stessa frecuenza di quella incislento, corrispon(lenti una al segno superiore (onda ordinaria) l'altra al segno inferiore (onda straordinaria) del doppio segno clue compare nelle (21), (23), proceslenti con velociti di fase e coefficienti d'assorbinento diversi, ricavabili separando la parte reale $n$ e quella immaginaria $z$ dellinsice di rifrazione complesso $n$. Tale separazione è molto complicata. e porta a formule di non immedisto significato; normalmente, si consiklerano dne situazioni tipiche, e precisamente quella della propagrzione quasi longitudinale $\left(\mathbf{B}_{H}\right.$ quasi parallelo alla direzione di propagazionc $w$ ) e quella della propaguzione quasi trasuersale (B $\mathbf{B}_{\text {if }}$ quasi perpendicolare alla direzione $x$ ), corrispondenti, in termini analitici, a trascurare nel raclicale della $(21)$ il termine $\mathrm{J}^{\mathrm{s}} / 1+(1-\mathrm{X}-i \not Z)^{2}$ rispetto al termine $Y_{L}$, o viceversa. Per quello che a noi importa, consitlereremo la sola propagazione longitudinale ( $Y_{T} \cong 0, Y_{L} \cong Y_{H}$ ), per la quale la (23), separanclo parte reale e parte immaginaria, clà:

$$
n^{2}=(n-i \not)^{-}=1-\frac{X^{r}}{1 \pm Y_{L}-i Z Z^{\circ}}
$$

\section{3. - TEOLIA DI STOREY.}

Tale teoria consiste nell'applicazione della (2.4) a pacelretti d'onde di bassa fiequenza, propagantisi nell'altia ionosfera, allo scopo di calcolarne la relocita di gruppo e quineli il tempo di propagazione lungo il tragitto (eventualmente contato più volte) inclivisluato sla una linea di forza del c.m.t.; caratteristiche della teoria sono certe approssimazioni semplificatrici.

La prima approssimazione deriva dal trasenrare, agli effetti rifrattivi e dispersivi, la parte di tragitto che si svolge nella bassa iono-

(*) Il simbulismu qui adottato d poraltro dive so ula quello della forsumla origiriale. 
sfera. Osserviamo ele la grandeza $Z=v / \omega$ decresce ropitamente con la quota (v ̀̀ dell'ordine di $10^{5} \mathrm{sec}^{-1}$ a $100 \mathrm{~km}$, th $10^{3} \mathrm{sec}^{-1}$ a $300 \mathrm{~km}$, di $1 \sec ^{-1}$ a $500 \mathrm{~km}$ ) e che l'apice rlella traiettoria rli un whishler è sempre a quota rilevante (cirea nor raggio terrestre per whistlers ricevuti a media latitudline); in prima approssimazione può dunque lecitamente assumersi $Z^{2} \ll 1$, e rlalla (24), separando la parte reale $\left(^{\left({ }^{2}\right)}\right.$, si ha:

$$
\begin{aligned}
& \text { a } \mid H^{2}=1-\frac{r}{1 \pm Y_{L}}-\underline{i}-\frac{f^{2} N}{f\left(f \pm f_{H} \cos \theta\right)}= \pm-\frac{f^{2} x}{f\left(f \pm f_{H}\right)}, \\
& \text { b } \quad \begin{array}{l}
R=\mp i, \\
\text { c } \quad S=0 .
\end{array}
\end{aligned}
$$

Dalle (25)e, (10) e (18)a si derluce $E_{x}=j_{x}=0$, cioè anclie il campo elettrico $\dot{e}$, in queste condizioni ai propagazione, trasversale. Talla (25)h si rleduce che le due componenti magnetoioniche clell'oncla sono polarizzate circolarmente in verso opposto.

Come si vede, la propugazione è dispersiva. J)alla (25)a, tramite la (4), si ricafa la velocità di fase 2 : la velocitì di gruppo,

$$
V=\frac{c}{u+f \frac{d n}{d f}},
$$

si otitiene ancora ralla (25)a:

$$
V=\frac{2 e\left(f \pm f_{H}\right)^{4 / 2}\left[f^{2}\left(f \pm f_{H}\right)-f f^{2} N\right]^{1 / 2}}{2 f^{3} \pm 4 f^{2} f_{H}-2 f f^{2} H 干 f_{H} f^{2} N} .
$$

Per i wlistlers si ammette:

$$
v \ll f<f_{H} \ll f_{N} ;
$$

è quindi, con accettabile approssimazione:

$$
1=\frac{3 e\left(\mp f_{H}-f\right)^{3 / 2} f^{1 / 2}}{f f_{H} f_{N}} .
$$

Osserviamo subito the $V$ i reale se $\mp f_{H}>-f$; risulta cosi clie per $i$ whistlers è possibile la propagazione della sola componente straorilinaria (segno inferiore rel rloppio segno), la eni velocità di gruppo vale, entro i limiti dell'approssimaxione accettata:

$$
V_{w}=\frac{2 c \sqrt{f\left(f_{H}-f\right)^{3}}}{j_{H} / N} .
$$


Se olu, con Storey, ammettiamo che lunfo tuto il tragitto di un wlistler sia

$$
p \ll f \ll f_{H} \ll f_{x},
$$

si puó serivere:

$$
V_{r v} \cong 2 e \frac{\sqrt{f f_{l t}}}{i_{N}}
$$

Ricordiamo, senza entrace in dettagli, rhe in un mezzo dispersivo e anisotropo, qual'è la ionosfera, si puó, con un procerlimento derivato dalla ben nota costruzione dell'indicatrice ottica dei cristalli birifrangenti, dedure la reluzione tra tirezione ti propagazione, normale d'onda e asse di anisotropia (qui coincidente con la lirezione del c.m.t.). Si trova, sempre ammottendo le (3I), che la direzione di propagazione giace tra la normale donda e la direzione tlel c.m.t., e ehe, inoltre, $V_{w}$ e puaticamente indipendente dalla direzione delli normale fionda anche per valori relativamente grandi li $\theta$ (sino a eirxa $\left.70^{\circ}\right)$. Costrutita l' indicatrice di raggion (cliagramma polar'e rli $\left.V_{t o}\right)$, risuita infine (11) che le direzioni di propagazione sono confinatu entro un cono (como di storey) avente per asse la direzione lel c.m.t. e semiapertura ri cilca $19^{\circ} 30^{\prime}$. In definiliva, le componenti a bassa frequenza degli atmosferici, incidendo, anche sotto angoli ragguardevoli (sino a cirea $70^{\circ}$ ) sulla bassa ionosfera, prenrlerebbero a propagarsi come componenti magnetoioniche stuardinatie, seguendo abbastanza strettamente linee di forza del e.m.t.

Il Lempo di propagnzione tw della componente di frequenza $f$ vale, come si ileduce dalla (32):

$$
t_{w}=\left.\left.\frac{1}{2 c \sqrt{f}}\right|_{i} \frac{f_{N}}{\sqrt{f_{H}}} d l \cong \frac{2 \cdot 10^{-10}}{\sqrt{f}}\right|_{i} \mid \frac{\lambda}{H} d l,
$$

essendo 7 il cammino seguito dal whistler e $I I$ l'intensitì del c.m.t. Saturalmente, componenti di frequenza diversa giungono al posto d'osservazione in istanti rliversi. Se si riportano, in un riferimento cartesiano, in ordinate le quantità $f^{1 / 2} \mathrm{e}$ in ascisse i corrispondenti istanti di ricezione, si ottiene una curva sensibilmente rettilinea, inclinata sull'asse delle ascisse di un angolo $a$; come senza difficolia si riconosce a partire ralala (33):

$$
\operatorname{cotg} a=D=\left.\frac{1}{2 c} \int_{i} \frac{f_{N}}{\sqrt{f_{H}}} d l \cong 2 \cdot 10^{-10} \int_{i}\right|_{H} \ddot{H},
$$


essendo // la cosickletta "dispersione" d(2l whistler osservato. Ha relazione ora seritta i molto importante in puanto mostra come sia possibile corralare, in un attendibile schema per l'andamento in quota ri $H$, rispersioni osservate e klensiti elet troniche lungo il camminn percorso: i whistlers si presentano così come un semplice mezzo d'indiagine prer ottenere informazioni sulla densitì elettroniea nell'esosfora, regrione inacessibile con i consueti metodi sli radiosonkagrios verticale lat suolo.

\section{1. - DEDE\%LNA F INTEGRAZIONI DELAA TEORLA DI STOREY.}

Come accennato, la teoria di Storey è stata ritoceata in varî punti per alattarla a successivi risultati sperimentali, e presisamente prr lav conto ai particolni tipi sli whistlers, quali i cosickletiti "nose whistlers", curntterizzati (blla contemporanea presenza di un segnale ¿li frequenza ascendente e uno di frequenza discendente rispetto aI tempo. Si rleve allo scopritore ali tali whistlers, R. A. Helliwell ("), losservazione che la $(2 \bar{T})$ nom possa in tali casi approssimarsi con la (32), e che vada invere consilerata la (30). Differenziando quest'ultima rispetto alla frequenza, si trova subito che la velociti e massima per la componente la cui frequenza vale $f_{n}=f_{n} / 4$ : ció spiega il caratteristico lligramma frequenza-tempo alei "nose whistlers ". Ha fresquenza massima, statisticamente piì elevata di quella llei whistler's normali, ¿̀ tlell'ortine di 10 liItz al ì genernlmente compresa nell'intervallo $1,5 \div 2,5 f_{n}$, corrispondente all'incirca all'intervallo $0,4-0,7 f_{n o}$, essendo $f_{n a}$ la girofiequenza all'apice della traiettoria: i " nose whistlers" damno cosi direttamente il valore di tale importante granklezza. Come senza difficoltà si deduce assumendo per il c.m.t. il cousucto morlello del campo di clipolo centrale, $f_{n}$ ì dell'orline di cirea $10 \mathrm{k} z$ per un whistler originatiosi a $45^{\circ}$ sli latitudine (apice a un raggio terrestre ali quota) e decresce rapiclamente all'aumentar. rella latitusline (circa $6 \mathrm{kH}$ ? a $60^{\circ}$ ): così, tali whistlers sono riceviloili soltanto a latitudine sufficientemente alta, e $i$ whistlers orlinari ricevibili a media latituline possono essere interpretati come il ramo inferiore di " nose whistlers " la eni frequenza caratteristica, $f_{n}$, è troppo alta $(*)$.

(*) Ja difficoltia di ricevere frequenze whistler relativamente ialle puć essere spiegata ricordando che per le componenti magnetoioniche siraordinarie l'assorbimento cresco rapiclamente via via cho $f$ s'avvieina a $j_{H}$. 
J. Tr. (). Storey si survì della (3t) per una stima della rensitáa elettronica nell'esosfera, assumendo costante il rapporto $N / I\left(^{*}\right)$ lungo la linea di forza del c.m.t. passante per il suo posto d'osservaziome (Cambrislge, Regno Cinito) e assumendo per $/$ ) il valor merlio di molte osservazioni; egli yervenue yer $N^{T}$ a un valore $\left(4.10^{\mathrm{B}}\right.$ elettomi a $\mathrm{m}^{3}$ a circa $12.000 \mathrm{~km}$ di (puota) molto piì grande del vero. Hiciamo subito che questa circostumza, imputahile essonzialmente al nom corteto metodo rli calcolo usato, non ì motivo sufficiente per rigettare la teoria di Storey nel siro insieme.

Ricortiamo che tale tworia riposa sn un'ipotesi, quella tlella propugazione longitudinule di omponenti stwordinarie a bassa frequenza della radioemissione associnta ad atmosferici, sulla quale non r'i motivo di avanzare grosse riserve, e su relazioni dolotite dalla troria magnetoionica per un plasma wniforme nell'ambito di parecchie Tumghezze d'onta e sotho te condizioni (31). Tiprendento il simbolismo usato precedentemente nel richiamare la teoria magnetoionica, le rourlizioni ora ricoriate possono essere scritte:

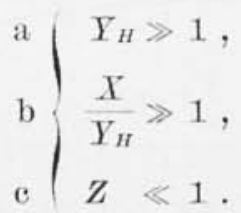

Sella Fig. 1. soun riportati schematicamente gli andamenti con la quota, in $\mathrm{km}$, delle grantlezze $X, Y_{H}, Z, X / Y_{H}$ per $f=10 \mathrm{kH} /$ (**) e $^{*}$ per propagazione strettamente longitudinale fla sorgenti a media latiturline. Attiriamo l'attenzione sul fatto che all'ajice della traiettoria viene generalmente meno la condizione (35)a, mentre nella bassa jonosfera vengono meno le rlue rondizioni (35)b e (35) $r^{2}$. La teoria tli Storey è runcule rriticabile per i seguenti the punti:

(A) il prospettrito mecennismo di magnetognirla (cono rli guida con apertura di circa $\left.19^{\circ} 30^{\prime}\right)$ non è efficace lungo tutito il tragitto;

(B) resta tla spiegre come i whistlers riescano a "perforare" cosi facilmente la bassa ionosfera, che per essi dovrebbe presentare,

$\left(^{*}\right)$ Ciò signifiea ammettere dhe $\mathrm{N}$ decrexea com il culw della quotat, se per $I I$ si assume un campo di lipolo.

(**) P'er $f<10 \mathrm{kllz}$, le grandezze $X, I, Z$ hanno valori proporzionaluente maggiori. 
sia per frugitti " dal basso" sia per tragitti "dall'alto", una rifleftivitic pintitosto alta.

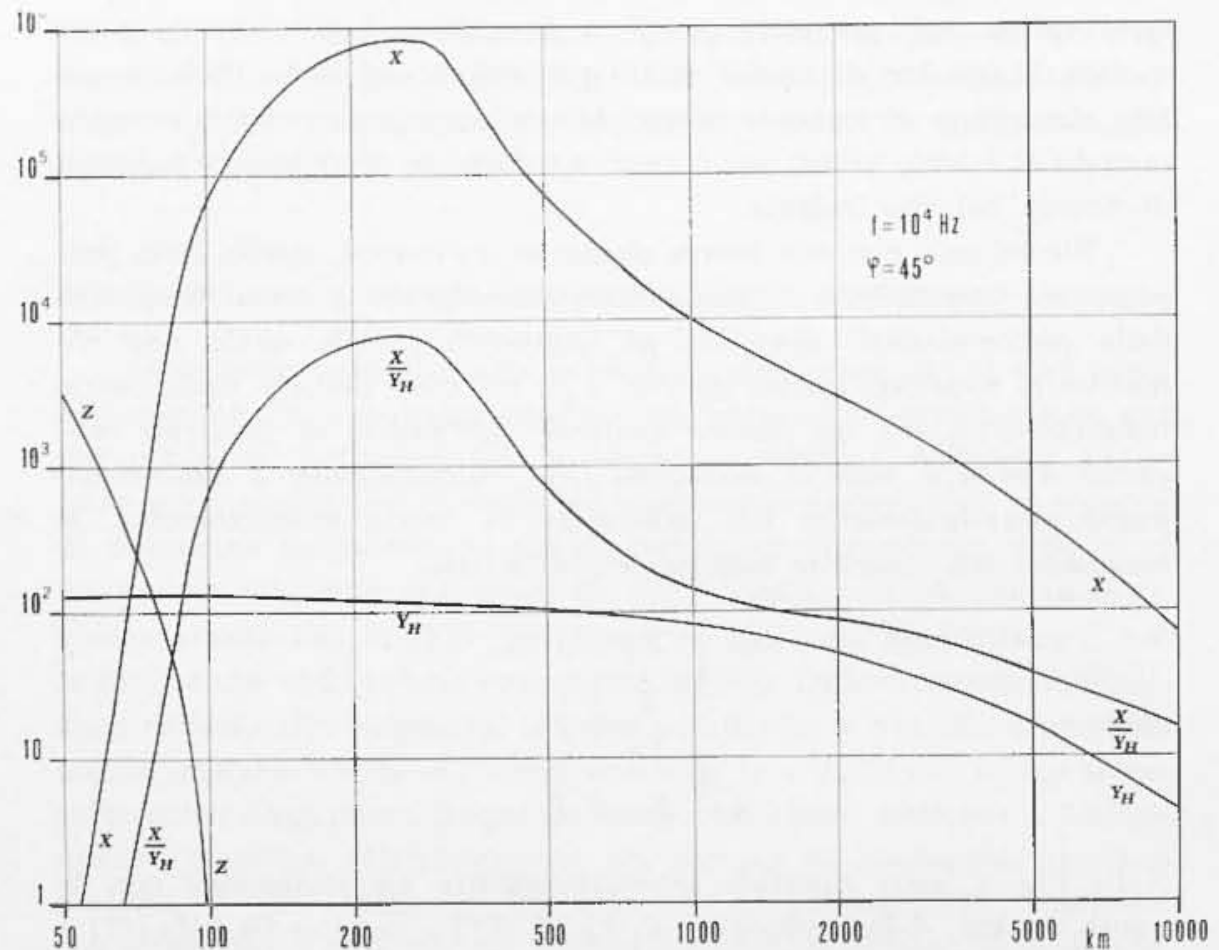

Fig. 1 - Andamento schematico con la quota delle granlezze $\boldsymbol{X}, \boldsymbol{Y}_{H}, Z$, $X / Y_{I I}$ per onde li froquenza $\left.10 \mathrm{k}\right] \mathrm{z}$ originate a $45^{\circ}$, li latidudine.

Jel punto (A), sul quale s'è appuntata lattenzione della maggior parte degli studiosi della questione, si può dare un'immediata giustificazione con un semplice ragionamento in termini di raggi di propagazione. Siano $a, b$ (Fig. 2) due linee di forza del c.m.t, abbastanza vicine, e $c$ un raggio di propagazione formante nu piccolo angolo $y$ con la linea $a$. Tndicando con $O_{\circ}$ la curvatura locale del raggio $c$, è, con riferimento agli elementi indicati in figura, $c_{o}=-d v / d s, d s=d r$ $\cos v \cdot r m \cos \vartheta$ - costante; svolgendio i necessari calcoli, $i$, a meno di infinibsimi di ordine superiore, $C_{o}=-n^{-1} d n / d r$. Nelle contizionti considerate, la propagazione is quasi longitudiunale, talclic vale la 
(25)a; dilterenziando quest'ultima, sostituendo nella precerlente re-

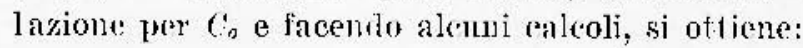

$$
C_{n}=\frac{1-n^{2}}{2 n^{2}} \frac{1}{N} \frac{d N}{d r} \mp \frac{f^{2} N}{f(f \pm f n)^{2}} \frac{1}{2 n^{2}}-\frac{d f_{n}}{d r} .
$$

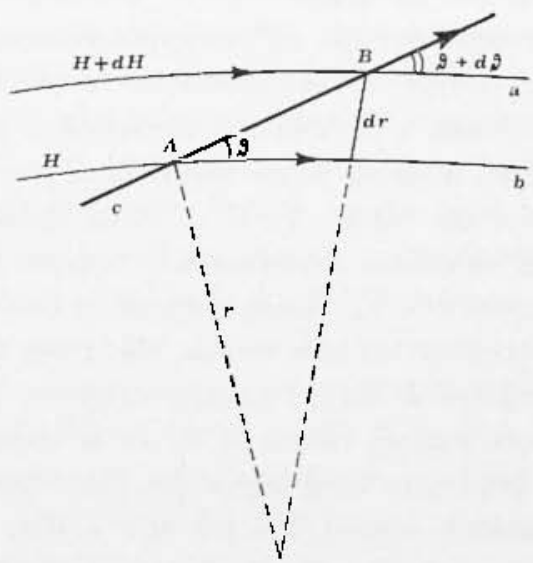

F'ig. 2

Quanto alla curvatura locale, $C_{n}$, delle linee di forza rel c.m.t., $\dot{e}$ $I J \cdot d \varphi==(H \div d I)(v+d v) d p$, siò̀, a meno di infinitesimi rli ordine superiore, $C_{n}=-\Pi^{-1} d I I / d r$; ricorlando la $(16) \mathrm{c}$, ̀े:

$$
C_{H}=\frac{1}{f_{n}} \frac{d f_{n}}{d r} .
$$

Se, con Storey, consirleriamo la ionosfera come un plasma uniforme nell'ambito della regione schematizzata nella Fig. 2 , si ha $d N / d r=0$, e se, ancora con Storey, ammettiamo le conrlizioni (35), per le componenti struortinarie risulta:

$$
C_{0}=-\frac{1}{2 n} \frac{f^{2} N f_{H}}{f\left(f-f_{H}\right)^{2}} C_{n} \cong-\frac{1}{9} C_{H}
$$

Per avere un eflett.o di magnetoguirla dev'essere però $C_{0} \simeq+C_{n}$, e dunque, accettaurlo le (35), non puó essere $d N / d r=0$ : così, se si accetta la teoria nella forma prima svolta, perehó si possa avere una propagazione magnetoguirlata deve esistere un appropriatio graliente trasversale rli ilensitia elettronica. 
Il risultato ilei semplice ragionamento ora fatto o confermato ta ralcoli piu complessi svolti da vari ricereatori; tipiei, al rigundo, i calenti di (G. Rao e II. G. Booker (\%). T) stata cosi proposta e sviluppata, frincipalmente ar opera di R. I. Smitl, R. A. Helliwell e I. IV. Yabroft $\left({ }^{10}\right)$, la cosilluteta teoria delle colonne di ionizzazione, secourlo la quale leltetto di magneloguira sistematicamente osservato ler i whistlers sarebbe clovuto allintrappolamento delle onde entro regioni ionizzate ("colomne") sviluppantisi lungo linee di forza del c.m.t.; in una "colonnan", la densita elettroniea avrebbe simmetria assiale rispetto a nua linea di forza magnetica, passando da un certo valore $N_{t}$ sullasse a un valore $N_{e}$ al di fuori della colomna, con $N_{e}$ minore (colomma ri massima ionizzazione) oppure maggiore (colonna. di minima ionizzazione) di $Y_{t}$. Senza entrare in dettagli, ci linitereno a ricordare, come risultati di tale teoria, che : per $1 / Y_{n}=f / f_{n}$ molto piccolo (diciamo minore di 0,1 ), l'intrappolamento delle onde avviene sempre (cioe per qualunque valore li $\theta$ ) se la rolomma è di massima ionizzazione feon gradiente comutrque piecolo; mai se la colomma d di minima ionizzazione; per $1 / \mathrm{Y}_{\|}$tra 0,1 e 0,5 , lintrappolamento puó avvenire in una colonna di massima oppure di minima ionizzazione purché il gratlente di ionizzazione sia non minore di un certo valore: per $1 / \boldsymbol{Y}_{n}$ tra 0,5 e 1 , l'intrappolamento puo avvenire soltanto in una colonna di minima ionizzazione, con appropriato gralientes.

Initerpretando rliscorsivanente i risultati ora ricorrati, accadrebbe clumque die alla sorgente, o eomunque nella bassa ionosfera, il whistler verrebbe intrappolato in una colonna di massima ionizzazione, anche tenue (siamo nel caso con $1 / Y_{U}$ molto piccolo), prenclendo a propusarsi lungo una linea ili forza ilei c.m.t.; procedendo lungo questa, la quautiti $1 / Y_{J}$ aumenterebbe jer la progressiva diminuzione di $f_{n}$ con la quota e la propagazione lungo quella linea proseguirebbe sinclé $1 / Y_{n}$ non superasse 0,5 e il gradiente trasversale di clensità (atetronica fosse abbastanza grande da soddisfare la condizione voluta lalla teoria (le componenti di alta frequenza, jer le quali $1 / Y_{n}>0,5$, verrebbero perse sotto forma di raggi deviati verso l'esterno delli (olouna); appena $1 / Y_{n}$ superasse il valore 0,5 , li propagazione lungo quella colomna cesserebbe, e i raggi verrebbero deviati verso zone con minore rlensità elettronica. Salta agli occhi il fatto che esisterebbe una frequenza di taglio, pari a $f_{17 a} / 2$, essendo, come già accemmato, $f_{n a}$ la girofrequenza minima, all'apice della traiettoria. Questo fatto nal s'accorla con la non infrequente ricezione di frequenze whistler nell'intervallo $0,4 \div 0,7 f_{1 / a}$ : tale ricezione potrebbe però spiegarsi 
ricorrendo it particolari mescanismi di propangazione (inflessione dei mggi verso quote più basse o sucessivo reintrappolamento nella (colonna).

Va osferrato clie la teoria delle colomne di ionizzazione i stata in sostanza claborata soltanto per integgrare la teoria magnetoionica in merito alla magnetoguida dei whistlers nell'alta ionosfera: essa infatti non apusure applicalile nellat bassa ionosfera e non d quindi in grado di dar conto del precedente punto (H).

Trn le detuzioni che si traggono dall'altuale teoria di propatgazione dei whistlers sono, come giì aceennato a proposito della teoria li Stores, informazioni sulla densitì eletironiea nell'nlta ionosfera; questi dinti s'ottengono, nell'ambito di un prescelto modello di distribuzione elettronien, merliante la (33) o, per i "nose whistlers", meriante l'analoga relazione ottenuta a partire dalla (30) anziché fistla (32). A J. H. Pope (1961) i dovuto un metodo di raleolo ( $\left.{ }^{6}\right)$, che appare assai più attendibile rli quello di Storey.

Tale metodo consistes sostanzialmente nell'integrazione rli nui forma differenziale derivata dalla $(30)$, in eni, mesliante l'introfluzione di un sistema rli coordinnte polari, la variabile d'integrazione is la latifurlina geografica; il pregio rlel metodo, pinttosto complesso, sta nel fatio che esso consente di verificarne a posteriori, sulla base dei risultati sperimentali (l'osservata dispersione dei whistlers), la correttezza rlellassunta funzione di distribuzione flella ciensitì elettronica e di determinare l'elTettiva linea di forza monetica seguita durante la propagazione (aho non è rletto coincida con quella passante per il luogo d'osservazione). L'applienzione tlel metodo porta ad assumere per la densità elettronica nell'alta ionosfera la funzione:

$$
N(r)=K^{2}(R / r)^{3} \operatorname{cxp}[3,03(R / r)],
$$

dove $r$ i la distanza dal centro tella Terra, $R$ il raggio teruestre, $K$ un fattore rli forma. I valori che per $N$ s'ottengono sono minori di quelli reriotti ra Storey, ma risultano aneora troppo grandi rispetto al vero: cio porta a sospettare che, il metorio di cnlcolo essenslo in sé corretto, la discortanza derivi tla un qualche errore sistematico insito nella (30).

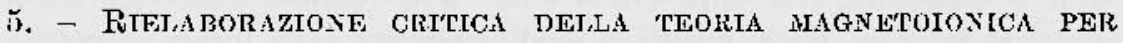 P'ROPAGAZIONE QUASI LONGIIUDINALE.}

Je considerazioni svolte nel precerlente paraguafo portano a ritenere che sia utile una revisione critica rella teoria magnetoionica, 
sia nelle jpotesi sia nello svolgimento, ral nomento the non ci si può ritenere a pirno soddisfatid all'integrazione di essa con la teoria delle colonne di ionizanione. Infatti, a parte le preche segnalate, per quest'ultima teoria c'e da segnalare l'evidente artificiosità. Si badi bene che con questo non si vool mettere in ulubbio l'ormai acertata anisotropia geomagnetian della listribuzione di elettroni e di altre particelle nellata atmosfera, e non si vuole neanche negare che meccanismi al magnetoguida sul tipo di quelli descritti dalla teoria possano aver luorgo. Quello che turba è la sistematicita di tali meccanismi, e dumque l'ammissione di urta distribuzione colomnare si rlensita elettronica ad hor per ogni whistler osservato. Is poichó la teoria in questione ha per seopo di accordare l'ipotesi di Storey con i risultati della teoria magnetoionica, ì su quest'ultima che in definitiva va posta un'attenzione critica.

Del resto, l'opprortunita di un riesame della teoria magnetoionica deriva anclue la altre circostanze sperimentali, oltre a quelle strebtamente legate a (puestioni di radiopropagazione magnetoguidata: ci riferiamo in particolare a esperienze sullinterazione tra onde nella bassa ionosfera ("), (lie hamno messo in evirlenza, tra l'altro, una insospettabile dipentenza della girofrequenza dalla densità elettronien, chiaro indice di un accoppiamento tra onde e.m. a plasma ionosferico ben piì complesso a quello consirlerato nella teoria magnetoionica corrente.

In merito alle ipotesi poste a base della teoria magnetoionica, un primo quesito che si pone è quello relativo all'introduzione o no, nell'equazione (11) dei moti eletronici, di un termine di " campo interso n, seguendo le linee della ben nota teoria di H. A. Lorentz sulla polarizzazione dei dielettrici non densi. Una sorldisfacente discussione di tale problema è stata fatta ila. J. A. Ratclilíe ( $\left.{ }^{8}\right)$, con la conclusione clue il termine in questione può essere lecitamente trascurato. Del resto, asserviamo noi, l'inclusione di tale termine porterebbe nella $(2+)$ semplicemente alla comparsa di certe grandezze numeriche, non alteranti funzionalmente la rifrativita ionosferica.

is stata cliscussa pure l'ipotesi (III), che è stata modifictuta in mosto da portare in conto, contemporaneamente, l'eftetto degli elettroni e rlegli ioni. Nell'approssimazione "longitudinale " in cui è stata ottenuta la (25)a, quest'ultima si molificherebbe nella forma seguente:

$$
n^{2}=1-\frac{f^{2} *}{f\left(f \pm f_{H}\right)}-\Sigma_{i} \frac{f^{2} N t}{f\left(f \mp f_{H i}\right)},
$$


essendo $f_{s i}$ e $f_{H i}$ la frequenza di plasma e la girofrequenza della $i$-ma specie cli ioni positivi presenti (ioni negativi vengono conteggiali ribaltando il doppio segno). Si da peraltro la circostanza che per oftenere un qualche elietto ali magnetoguich dovuto anche a ioni, questi dovrebbero avere ma rensiti molto maggiore di quella degli rlettroni: mallora in genere carlrebbe lipotesi (I) e non sarebbe più corretta lat teoria nel suo insieme.

In merito all'interazione eletloni-particelle circostanti, H. K. Sen e A. A. Wyller ("), e sulla loro scia altri, hamo rielaborato la teroria sostituendo all'ipotesi $\left(\mathrm{IV}^{\mathrm{Y}}\right)$ mo schema piì complesso, nel quale la frequenza $y$ delle collisioni viene assunta come una funzione lineare del rapporto tra l'energia cinetica degli elettroni e l'energia di agitazione termica velle particelle circostanti, e pro l'energia degli cletironi viene assunta ma distribuzione quasi maxwelliana. Tale teoria nota (oon il nome, in realtà alquanto enfatico, di "teoria magnetoionica. generalizzata n, non porta, al onta della notevole complessità dei calcoli, a rilexanti modifiche funzionali per la rifrattivitit: se $f \gg v$ valgono ancora le formule "classiche", e altrimenti basta moltiplicare in queste ultime $v$ per un opportuno coefficiente numerico, variabile tra 2,5 (se $v<\left|f-f_{H}\right|$ ) e 1,5 (se $v>\left|f-f_{H}\right|$ ).

$A$ questo punto, non avendo ricavato grosse conseguenze dal riesame rlelle ipotesi a base della teoria magnetoionica, non resta che velere se eventuali conseguenze si hanno non effettumblo nei alcoli essenziali della teoria cute apurossimazioni, la noi prima esplicitamente indicate ma che normalmente non vengono enfatizzate: ci riferiamo al trascurare l'induzione $B$, lel eampo dell'onda rispetto all'iuluzione $\mathbf{B}_{n}$ rlel c.m.t., e alla trattazione euleriana dei moti elettronici suscitati ralle onde incidenti.

('osi, mantenendo il formalismo dianzi introriotto, riscriveremo la (11) nel modo seguente:

$$
m \ddot{\mathbf{r}}=q \mathbf{E} \div q \dot{\mathbf{r}} \times\left(\mathbf{B}+\mathbf{B}_{n}\right)-m \boldsymbol{u} \dot{\mathbf{r}}
$$

Poniamo ora, come è sempre possibile fare,

$$
\dot{\mathrm{r}}=\dot{\mathrm{r}}+\dot{\mathrm{s}}
$$

love $\mathbf{r}$ è la velocitì di scorrimento degli $N$ elettroni per unità di volume, legata alla densità della comente di conduzione j dalla (12). Hediando la (42) per gli $N$ elettroni nell'unità di volume, è chiara- 
mente $\overline{\mathbf{s}}=0$, mentre per le accelerazioni, nulla potenclo dire al momento, scriveremo

$$
\ddot{\mathrm{r}}-\frac{d}{d t} \dot{\mathbf{r}}+\ddot{\mathbf{s}}=\frac{i}{N_{q}}\left(\because \frac{k}{N_{q}} j_{x}\right) \mathrm{j}+\ddot{\mathbf{s}}
$$

Lit (41) dì cosi:

$$
m \frac{i \omega}{N q} \mathbf{j}-m \frac{i k}{N^{2} q^{2}} j_{x} \mathbf{j}+m \ddot{\mathbf{s}}=q \mathbf{E}+\frac{\mathbf{1}}{N} \mathbf{j} \times\left(\mathbf{B}+\mathbf{B}_{H}\right)-m \frac{v}{N_{q}} \mathbf{j} .
$$

Moltiplieando tutto per iNq/mo, utilizzando le posizioni della Tab. IT a ponendo ulteriormente

$$
\begin{aligned}
& \mathbf{Y}=\frac{\pi \dot{q}}{m \omega} \mathbf{B}, \\
& \mathbf{W}=\frac{N q}{\omega} \ddot{\mathbf{s}},
\end{aligned}
$$

si ha infine l'equazione vettoriale

$$
\cdots-\mathbf{j}+\frac{b}{N(\omega)} j_{x} \mathbf{j}=i \varepsilon_{o} \omega X \mathbf{E}+i \mathbf{j} \times\left(\mathbf{Y}+\mathbf{Y}_{H}\right)-i Z \mathbf{j}-i \mathbf{W}
$$

che dì luogo alle tre equazioni scalari:

$$
\begin{aligned}
& \text { a } \quad-j_{x}+\frac{k}{N(\omega \omega} j_{x}^{2}=i \varepsilon_{0} \omega X D_{x}+i j_{y} Y_{z}-i j_{z} Y_{y}-i j_{z} Y_{T} \\
& -i \not j_{x}-i W_{x} \text {, } \\
& \mathrm{b}-j_{y}+\frac{k}{N_{q} \omega} j_{x} j_{y}=i \varepsilon_{0} \Leftrightarrow X E_{y}-i j_{x} Y_{z}+i j_{z} Y_{\mathbf{L}}-i \not j_{y} \\
& \text { e } \begin{aligned}
& -i W_{y}, \\
-j_{z}+\frac{k}{N q \omega} j_{x} j_{z} & =i \varepsilon_{\theta}(t) X E_{z}+i j_{x} Y_{y}+i j_{x} Y_{z^{\prime}}-i j_{y} Y_{L} \cdot- \\
& -i Z j_{z}-i W_{z} .
\end{aligned}
\end{aligned}
$$

Potremmo risolvere questo sistema di equazioni seguendo una via simile a quella seguita nel preceilente paragrafo 2, la soluzione contenendo naturalmente le ulteriori grandezze incognite $W_{x}, W_{y}$, $W_{z}$. Poiché peraltro abbiamo polarizzato l'attenzione sulla magneto- 
gnicla, ci limiteremo a una trattazione molto piǹ semplice, consicłerando una propagazione quasi longitudinale $\left(Y_{T} \cong 0, Y_{L} \cong Y_{H}\right)$. Sotto questa ipotesi, ricorlando le ( $\tau),(8)$, (9) e la (2), potremo scrivere:

$$
\begin{aligned}
& \text { a } i W_{x}=(1-X-i Z) j_{x}-\frac{n}{N_{\eta} c} j^{\mu_{x}}+\frac{q n(1+R-)}{\varepsilon_{0} m c m\left(n^{2}-1\right)} j^{2} y \text {, } \\
& \text { 1) } i W_{y}=-\left(1 \div \frac{\mathrm{I}}{n^{2}-1}-i R Y_{L}-i Z\right) j_{y}- \\
& -\frac{n}{N_{q} c}\left(1+\frac{X}{n^{2}-1}\right) j_{x} j_{y}=A j_{n}-\left(\dot{r} j_{z} j_{y},\right. \\
& \text { o }\left\{W_{z}=\left(1+\ldots \frac{X}{n^{2}-1}-i \frac{Y_{L}}{R}-i \%\right) R j_{H}-\right. \\
& -\frac{n}{\lambda_{i \eta c}}\left(1+\frac{X}{n^{2}-1}\right) R j_{n} j_{u}=\theta j_{y}-\left(\cdot R j_{x} j_{H}\right. \text {. }
\end{aligned}
$$

I)alle due ultime equazioni risulta:

$$
R=\frac{A-\left(1+\frac{X}{n^{2}-1}-i Z\right)}{i T_{t}}=\frac{\left(t+i Y_{L}\right.}{1+\frac{X}{n^{2}-1}-i Z}
$$

ì quindi:

$$
1-\frac{X}{n^{2}-1}-i Z=\frac{A \pm \sqrt{A-4 i G_{L}+4 Y_{L}^{2}}}{2} \text {. }
$$

In virtì della struttura delle $(19) \mathrm{b}$, (49)c, appare ragionevole ammettere che sia $W_{z}=R W_{n}$; segue allora, dalle medesime equilzioni, che is $G=R A$, e ancora, con calcoli immediati:

$$
1+R^{2}=0
$$

Questa relazione è la (25)b della teoria elassica, esprimente la polarizzazione circolare delle componenti ilell'onda nel piano $y z$, ortogonule al campo magnetico di guila; aver ritrovato questa circostanza, ben verificata sperimentalmente, costituisce una buona prova dell'attendibilita dell'ipotesi espressa dalla relazione $W_{z}=R W_{y}$. 
Introulueendo la (52) nella (49)a si ha:

$$
j_{x}=\frac{N(\underline{e}}{2 n}|(1-X-i Z) \pm(1-X-i Z)| 1-\frac{\sin W_{x}}{N\left(g e(1-X-i Z)^{2}\right.} \mid .
$$

Tenendo conto della $(46)$, si riconosee che il radionle i lecitamente ajprossimalbile eon l'unità; segue:

$$
\begin{aligned}
& \text { a } j_{x}=0 \\
& \text { b }\left\{j_{x}=\frac{N_{2} r}{i i}(1-X-i Z)\right. \text {. }
\end{aligned}
$$

Facendo $(t=R .1$ nella $(\bar{n} 1)$ si ottiene poi $(*)$ :

$$
n^{2}-1=\frac{2 X}{A \pm\left(A \mp 2 Y_{L}\right)-2+2 i Z} .
$$

Prendendo il meno del primo doppio segno si ha:

$$
n^{2}=(n-i \chi)^{2}=1-\frac{Y}{1+Y_{L}-i Z}
$$

separnato parte reale e parte immaginaria:

$$
\begin{aligned}
& \left.\left.\chi^{2}\right\}^{(* *)}=\frac{1}{2}\right\} \pm\left[1-\frac{\mathrm{Y}\left(1 \pm Y_{L}\right)}{\left(1 \pm Y_{L}\right)^{2}+Z^{2}}\right]+
\end{aligned}
$$

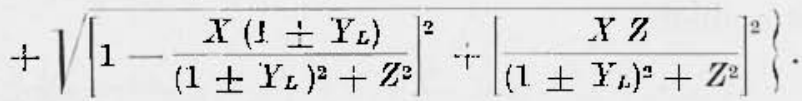

Prendendo nella (5̃) il più del mimo doppio segno si ha invece:

$$
n^{2}=1-\frac{Y}{1-A \pm Y_{L}-i Z}
$$

Posto:

$$
\tau=i \frac{W_{y}}{j_{y}}
$$

(*) Qui e nel segnilu seriverento il sloppin segno relativo a $Y_{L}$ in accordo con Ia (25)l), in modo whe il segno superiore si riferisca alla componente ordinaria.

(**) $n^{2}$ corrisponde al +. del primo doppio segno; gli altri doppi segni rignardano lo due eomponenti magnotoioniclie. 
dalla $(49) \mathrm{b}$ e (54)a si ha $A=E$, e dalla (58) segne:

$$
n^{2}=1-\frac{X}{1-U ! Y_{L}-i Z}
$$

sempre dalla $(19)$ b, ma stavolta con la $(5-1) b$, si ha:

$$
n^{2}=1-\frac{X^{2}+i X Z}{X-U \pm Y_{L}} .
$$

La grantezza $U$ è legata alla dinamica ciei moti clettronici; precisamente, richiamanclo la (f6) c altre relazioni precerlenti, si può serivere:

$$
U=i \frac{N q}{(t)} \frac{\ddot{s}_{y}}{j_{y}}=-\frac{N q}{\frac{\partial j_{y}}{\partial t} \ddot{s}_{y}} \cong-\frac{\ddot{s}_{y}}{\ddot{r}_{y}}
$$

Le due componenti di accelerazione che compriono nel rapporto ora seritto riguardano rispettivamente il contributo del moto di ciclotrone, con raggio en, e del moto di scorrimento, con raggio o, al risultante moto medio elettronico nel piano ortogonale al campo magnetico di guida; $U$ risulta pari, in valore, al rapporto $\varrho_{H} / \mathrm{e}$, clle, a sua volta, risulta pari ( $\left.{ }^{(}\right)$a $1 / Y$. Risulta così clıe, nel campo in cui $Y \gg 1$, ̀े $U \ll Y$.

Trascurando dunque $U$ rispetto a $Y_{L}, l_{k}(60)$ si rifluce alla $(56)$, mentre dalla (61) si lia:

$$
n^{2}=(n-i z)^{2}=1-\frac{X^{2}+i X \not}{X \pm Y_{L}} ;
$$

separando parte reale e parte immaginaria:

$$
\left.\left.\begin{array}{l}
n^{2} \\
\chi^{2}
\end{array}\right\}^{(*)}=\frac{1}{2}\left\{ \pm\left(1-\frac{X^{2}}{X \pm Y_{L}}\right)+\sqrt{\left(1-\frac{X^{2}}{X \pm Y_{L}}\right)^{2}+\left(X \pm Y_{L}\right.}\right)^{2}\right) .
$$

b. - DISCUSSIONE DEI RISULTATI.

(a) Le equazioni (-19) mostrano che, accanto a termini lineari. già presenti nelle (18) della teoria classica, compaiono termini di se-

(*) $n^{2}$ enrrisponde al + del primo doppio segno; gli altri doppi segni riguardano lo due componenti magnetoioniche. 
conclo suado per le componenti di corrente e quindi, a norma delle (s), per lo componenti di campo eletrico. Tali termini nou lineari derivano dalladotiata rappresentazione delle velocità elettroniche

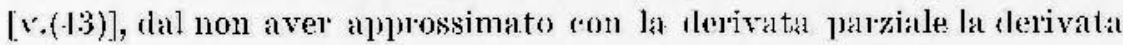
temporale delle velorita. elettroniche [v.(44)], dall'aver considerato anwhe il campo magnetico dell'ouda [v.(4) $]$. E importante osservare che, rome senza difficolta si verilica, tali temini svaniscono se si consislera uno solo dei procedimenti ora ricordati, per gli altri due adottandosi le approssimazioni usuali: essi rappresentano innque un effotto rooperativo d"accoppiamento non lineare e dispersivo tra onile e mezzo, qualitativamente esprimibile dicendo whe le onde modificano rifletlivita e assorbiviti del mezzo, in modo dipendente dall'intensità e dalla frequenza. Si la l'impressione che le teorie elaborate per dar conto di certi fenomeni non lineari nella radiopropagazione ionosferia [ali cui un'eceellente rassegna is il riferimento bibliografico (i)], attualmente piutosto starate dal corpo della teuria magnetuionica, possanu trovare la loro sistemazione nell'ambito di una teoria magrnetoionica generale sviluppata seconto le linee schematiomento indicate sopra. P'er quanto estremamente interessante, tale questione esula peró dal limitato scopo del presinte lavoro.

(b) Il mantenimento della polarizzazione circolare delle onde in un piano ortogonale al eampo magnetico di guida [v.(52)], porta alla scomparsa del termine in $j^{2}{ }_{4}$ nella (49)a; l'altro temine non liteare, in $j^{2}{ }_{x}$, porta ai valori (ó4) jer $j_{x}$. Joi questi valori, il primo, nullo, is coerente con la teoria classica, mentre il secondo, che rivela una componente longitudinale di corrente e di campo elettrico, sta a significare che le traiettorie elettroniche hanno anclamento clicoislale (nei tratti tra una collisionc e l'altra) intorno alla direzione del campo magnetico tli guisla.

(c) La (56) è la (24) della teoria classica. Par la parte di tragitio non svolgentesi nella bassa ionosfera, per la quale si puó ammettere $Z^{3} \ll 1$ (all'incirca, al thi sopra della regione E: Fig. 1), la parte reale ilella (56) dí luogo alla (25)a; come s'ì visto a sio tempo, ammettonclo le limitazioni (28) puó propagarsi la sola componente straordinaria, con velocití di gruppo data dalla (30) ovvero, con le più restrittive limitazioni (3.1) di Storey, tata lalla (32), la quale ultima dii poi, per la dispersione, la (34). Il fatto che quest'ultima relazione sia in buon accorda con l'esprerienza porta ad accettare l'applicalbiliti 
lelle relazioni ora ricordate per l'anzidedta paute sli traiettoria, clue i poi l'infera traietioria as esclusione alel tatio mela bassa ionosfera: qui la (3-1) carle in difetto per il non contemporaneo verificarsi delle comblizioni $Z^{2} \ll 1$ e $x / Y_{n} \gg 1$. S'e visto pure che per spiegare la magnotoguila ileva esistere un appropriato gradiente trasversale di rlensita eletronica, il che ha portatu all'ipotesi di colome di ionizzazione rentrate su line di forza del campo magnetico ferrestere. V'é da ritenere peraliso elie il riscontauto effetto si magnetugruida possa essere spiegato sulla base della corrente lungitudinate ricorkata nel precelente punto (b).

In efletii, posto clat, in virtì delle $(9)$ e (52), la rlensità di currentie trasversale i nulla, la corrente netta suscitata dallonda è puramente lungrituslinale; eio accade evislentemente prer la partioblare ronformazione del campo elettrico, ma nulla viela di rapjresentare le cose sostituendo al phasma reale un plasma littizio caratterizzato da ma Ilensità elettroniea $N^{\prime}$ anisotropa, non nulla netla slirezione dol campo magnetico e nulla in direzioni ortogonali. E cinesione oprinabile, e romunque non particolarmente importante per la presente discussione qualitativa, la forma da assegnare alla funzione $X^{\prime \prime}(0)$; sta li fatto (rhe in 111 molello del genere s'avolobe un gradiente trasversale di densità dettronica piuttosto grande (teoricumente, infinito), tale sla assicurare, fatta salva la condizione $f \leqslant f_{n} / 2$, la guicla intorno a linee di forza magneticle (*). La modifica improsta dalle onle al mezzo, eui s'è aceennato nel precenlente punto (a), viene giai interprebata come la fulmazione di una columa di massima ionizzazione, allineata con il campo manetico di guisla. Ia teoria delle colonne di ionizzazione risulta cosi ancoratn alla teoria magnetoionica, costituendu unu ‘lei risultati di essa.

(11) Passando ura alla (63), osserviamo preliminarmente clie questa relazione dà conto ilellesistenza di altre lue componenti magnetoioniche, clie eliameremo componenti modificate, aventi in comune con cuclle "classiche" la polarizazione circolare in versi ofrposti in un piano ortogonale al eampo magnetico sli gnirla. Per valutare l'importanza di queste compromenti, risjetio a quelle classiclıe, convernì

(*) Ovviamente, prestendo per bunti i risultati della terria velle co. lmme di ionizzazione. 
considerare, per semplicità, il caso in eui sia $\bar{Z}^{2} \ll 1$; la parte reale della (63) dit allora:

$$
\begin{aligned}
& n^{2}=1-\frac{X^{2}}{X \pm Y_{t}}=i-\frac{X}{1 \pm \frac{Y_{L}}{X}}=i-\frac{f^{2}{ }_{N}}{f\left(f \pm f^{\prime}{ }_{n} \cos \vartheta\right)} \cong \\
& 1-\frac{f^{\prime 2}}{ \pm\left(f \pm f^{\prime}{ }^{\prime}\right)} .
\end{aligned}
$$

Confrontando con la (25)a, si vede che le cose vanno come nella teoria classica, a patto cli considerare una "girofrequenza modificata"

$$
f_{H}^{\prime}=\frac{f^{2}}{f^{2}} f_{H},
$$

che dì conto, sotto un diverso aspetto, del complesso accoppiamento tra onde, plasma e campo magnetico. It interessante osservare che tale girofreqnenza è direttamente proporzionale al quadrato della frequenza $f$ delle onde e inversamente proporzionale al quadrato della freqnenza di plasma $f_{N}$, cioè inversamente proporzionale alla dlensitá clettronica $N$; enineirle con la girofrequenza $f_{I}$ per $f=f_{N}(X=1)$. Tale dipendenza della girofreqnenza mollificata da $N$ potrebbe spiegare, nei casi in eni si può ammettere l'esistenza delle componenti magnetoioniche modilicate (cioè, come vedremo, nella bassa ionosfera), la variazione della girofrequenzil eftettiva osservata in alcune esperienze sull'interazione di onde (1). Tornando alla (65̃), le due componenti anzidette esistono, o per dir meglio si propagano, se $\|$ è reale. Se $X \gg Y_{t}$, per avere $n$ reale occorre che sia $X \leqslant 1$; ma queste due condizioni non si verificano mai insieme nell'ordinaria propagazione whistler, in cui è $Y_{L}>1$. Escluso dunque il caso $Y_{L}<X<1$, per la componente ordinaria $n$ è reale se $X^{2}<X+Y_{L}$, annullandosi (riflessione) per $X^{2}=X+Y_{L} ;$ se, come accade per i whistlers nella bassa ionosfera, $Y_{L} \gg 1 / 4$ (Fig. 1), la propagazione della componente ordinaria è possibile, a conti fatti, soltanto per $X<\sqrt{\Gamma_{L}}$. Quanto alla componente straordinaria, si trova analogamente che $n$ è reale se $X<Y_{L}$, ì infinitamente grande per $X=Y_{L}$ e non s'anmulla mai.

Risnlta dnnque che le componenti modificate hanno notevole rilevanza nella bassa ionosfera, e che di esse interessa principalmente la componente straordinaria. Nella bassa ionosfera, peraltro, non is $Z^{2} \ll 1$, come per semplicità ammesso; occorre pertanto tener conto dell'eftetto rifrattivo delle collisioni clettroniche, ciò̀ utilizzare le (5T) e (64) così come sono. Per studiare l'andlamento delle funzioni 
$n^{2}\left(X, Y_{H}, Z\right)$ e $\chi^{2}\left(X, Y_{H}, Z\right)$, date dalle relazioni ora ricordate, abbiamo pensato di far ricorso alle curve rappresentative delle funzioni

$$
\left.\begin{array}{l}
n^{2} \\
\chi^{2}
\end{array}\right\}=\frac{1}{2}\left\{ \pm(1-\zeta \xi)+[1+\zeta \xi(\xi-2)]^{1 / 2}\right\},
$$

parametriche in $\zeta$, mostrate nella Fig. 3 , che J. A. Ratclilfe $\left(^{8}\right)$ ha tracciato, ponendo $\xi=\mathrm{K}$ e $\zeta=\left(1+Z^{2}\right)^{-1}$, nel cuso $Y_{H}=0$ (assenza di
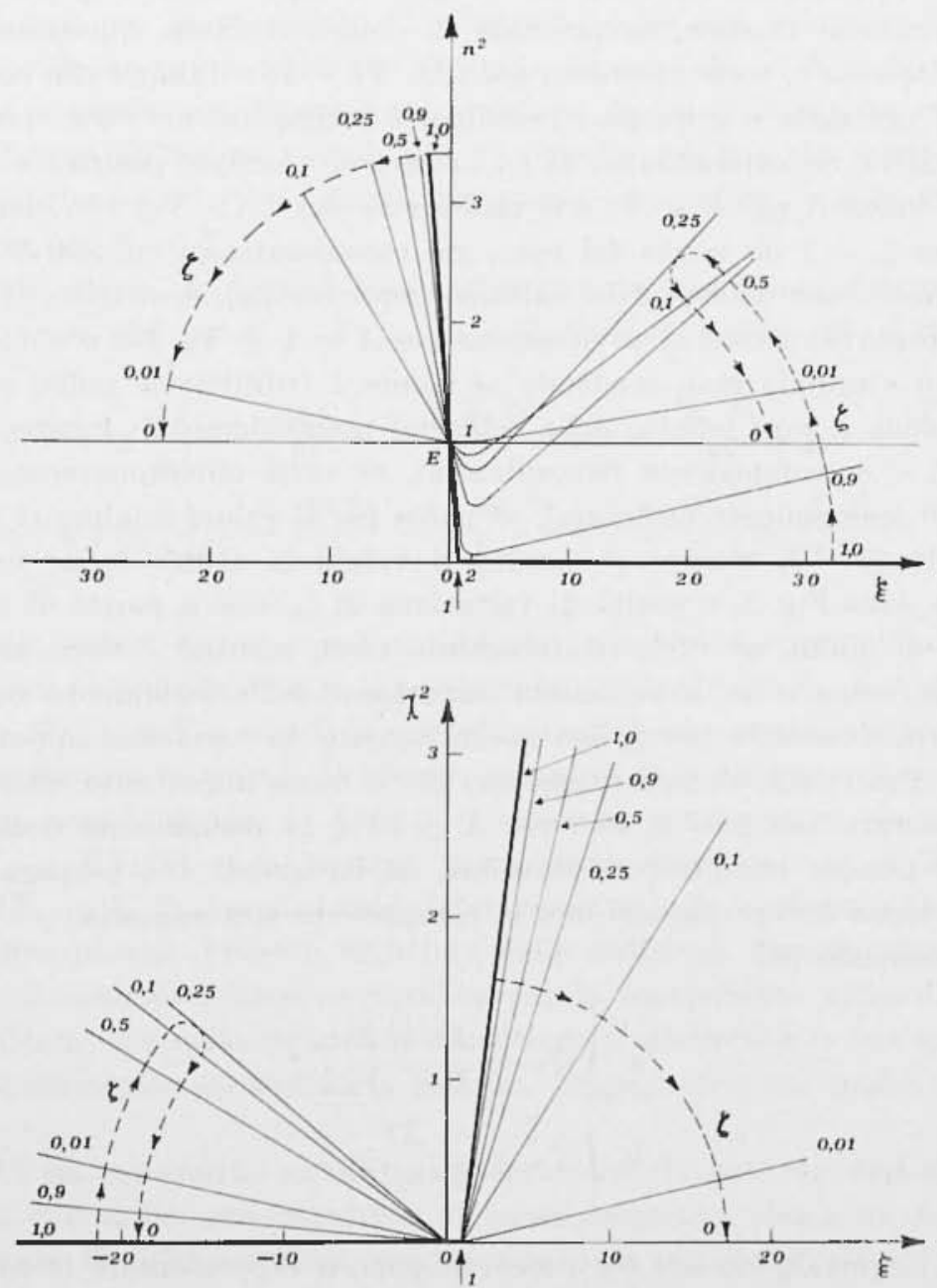

Fig. 3 - Curve di dispersione per un magnetoplasma assorbonte. 
campo magnetieo), ma che, a nostro parere, hamo un utilitio pinttosto gentrale. Infatii, ponemelo

$$
\begin{aligned}
& \text { a } \quad \xi_{c}=\frac{1}{1 \pm Y_{L}}, \\
& \text { b } \zeta_{c}=\frac{Y_{\gamma_{H}}}{Y_{2_{2}} \div \gamma^{2}} \text {, }
\end{aligned}
$$

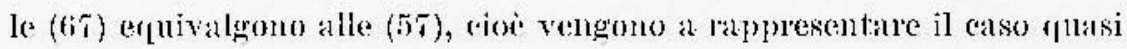
longiturlinale rassico, in presenza di collisioni. Nella questione che fui interessa $\dot{c}$, come ricordato poco fa, $\zeta_{L} \gg 1$, e clunque alla componente ordinaria e a quella stramerilinatia corrispondono valori positivi

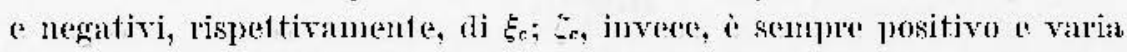
tra il valore 1 per $Z<J_{L}$ e il valore zero per $Z\left(>J_{L}\right) \rightarrow \infty$. Sal che

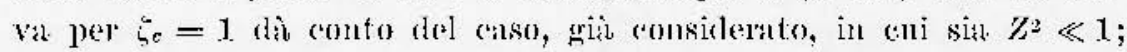
in questo cuso (efletto delle collisioni trascurabile), i $n^{2}\left(\xi_{c}=1\right)=0$, cioc, come satppiamo, si lia rillessione per $X=1 \pm J_{s}$. Per $0 \leqslant \zeta_{c}<1$,

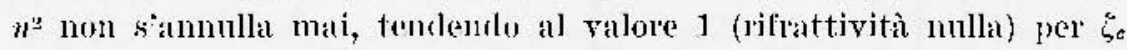
che temile a zero (elfefto alelle collisioni preponderante); mentre pero per $\varepsilon_{c}<0$ (componente silaurlinaria), "n2 varia monotonamente, per $\xi_{n}>0$ (componente ordinaria), $n^{2}$ passa per it valore minimo $\left(1-\varepsilon_{r}\right.$ ) fuando $\xi_{c}=2$, mentre $\chi^{-}$assume il valore $\xi_{r .}$ ('om'e facile vedere,

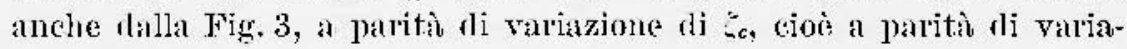
zione li quota, $n^{2}$ varia relativamente poco, mentre $\chi^{-}$varia ampiamente; come si sa, a variazioni importanti dell'assorbimento su to'igitti relativamente brevi, non accompagnate rla variazioni importanti lella rifrattivitio, s'acompryona mut piǹ o mono imprortante riflessione

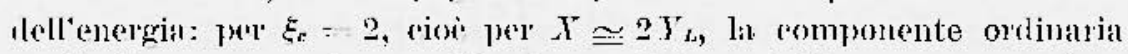
perte dungue energia per piflessione, ed ha quindi una propanazione assai mono favorevole che non la componente straordinaria.

Ponenrio poi

$$
\text { b }\left\{\begin{array}{l}
\xi_{m}=\frac{X^{2}+Z^{2}}{X \pm Y_{L}}, \\
\zeta_{m}=\frac{X^{2}}{X^{2}+Z^{2}},
\end{array}\right.
$$

le (bit) equivalgono alle (6i-t), rioe vengono a rapleresentare le componenti molificate in presenza di collisioni. Il partunetro $\zeta_{m}$ is sempre positivo, e varia tra il valore 1 pes $Z<X$ (effetto delle collisioni tra- 
scurabile) e il valore zero per $Z(>X) \rightarrow \infty$. Quanto a $\xi_{n}$, esso is positivo per le due componenti se $X>Y_{L}$, mentre se $Y<Y_{L}$ ì positivo per la sola componente ordinaria. Quando $\xi_{m}$ è positivo, la situazione è analoga a quella vista poco fa per la componente ordinaria classica, nel senso che si ha una più o meno importante rillessione per $\xi_{m}=2$, cioè per $X^{2}+Z^{2}=2\left(X \perp Y_{h}\right)$; valeudo questa condizione, per $X \geqslant Z$ si ottiene la (65), per $X<Z$ si ha $n^{2}=1-\left(X^{3} / Z^{2}\right)$. All'impossibilità della propagazione della componente ordinaria per $X>Y_{L}, X \geqslant Z$, già trovata precedentemente, discutencio la (65), s'aggiunge ora la possibilità di riflessione delle due componenti nell'intervallo di quote, piuttosto ristretto, in cui è $Y_{L}<X<Z$, molto vicino a quella che chiameremo "regione di transizione", in eni $X$, $Y_{L}, Z$ sono all'incirca uguali (Fig. 1). Risulta dunque che il morlo ti propagazione favorito $\dot{e}$, anche per queste componenti, quello straordinario per $X<Y_{L}$.

Alle quote alle quali l'effetto rifrattivo delle collisioni elettroniche è rilevante, ciò̀ per $X<Y_{L}(\gg 1)<Z$, dalle $(6 \pi)$, (68), (69) si ricava:

$$
\begin{aligned}
& \text { a } \quad n^{2} \cong \frac{1}{2}\left\{\left|1+\frac{X^{3}}{Z^{2}}+\frac{X Y_{L}}{Z^{2}}\right|^{1 / 2}+1+\frac{X Y_{L}}{Z^{2}}\right\} \cong 1+\frac{X Y_{L}}{Z^{2}}, \\
& \text { b } \quad n^{2} m \cong \frac{1}{2}\left\{\left|1+\frac{X^{2} Z^{2}}{Y^{2}{ }^{2}}+2 \frac{X^{2}}{Y_{L}}\right|^{1_{1 / 2}}+1-\frac{X^{2}}{Y_{L}}\right\}=\frac{X Z}{2 Y_{L} .}
\end{aligned}
$$

E facile vedere che $n \div$ e $n_{m}{ }^{2}$ si ridueono, con buona approssimazione, al valore dato dalla (25)a se si consilerano, in luogo di $\Gamma_{L}$, le quantitit $Y_{c}^{\prime}-Z^{2} / Y_{L}$ e $Y^{\prime}{ }_{n}-2 Y_{L} / Z$, rispettivamente. $\Lambda$ questo punto, possiamo riferirci ai calcoli di Storey, nei quali la rondizione di validitì per la magnetoguida è $X / Y \gg 1$ : per la componente classica ì $X / Y^{\prime}{ }_{0}=X Y_{L} / Z^{2} \ll 1$, mentre per quella modificata i $X / Y^{\prime}{ }_{m}=$ $\mathrm{IZ} / 2 Y_{L} \gg X / Y_{t}$. Concludendo sinteticamente, nella bassissima ionosfera, dove prevale l'effetto rifrattivo delle collisioni, l'intrappolamento entro il cono ali Storey avverrebbe per la componente straordinaria modificata, che nella regione di transizione trasferirebbe la sta energia alla componente straordinaria elassiea, propagantesi nel morlo dianzi visto.

Ci sia consentita un'ultima osservazione. Fे noto da varì esperimenti con radioonde impulsive di bassa frequenza che, a un segnale incirlente $W$ sulla ionosfera, corrispondono un segnale rifratto (il " modo wlistler ", cioè, come abbiamo visto, la componente straordinaria) e due segnali riflessi, uno tei quali, $C$ (" eco d'accoppiamento $n)$ lith 
la stessa polatrizazione del segnale primario, mentse laalto, $P$ a eco principale m) la nua polarizzazione anomala rispolto a quela determinabile ron la feoria magnetoionica classicu. La spiegazione corrente dii cii, secondo mo'ipotesi (ipotesi drll'arcoppinnento) emessa anni fil lia R. MV. Parkinson $\left(^{5}\right)$, è schematizzala nelle Fig. 4 , a sinistra. Menlre
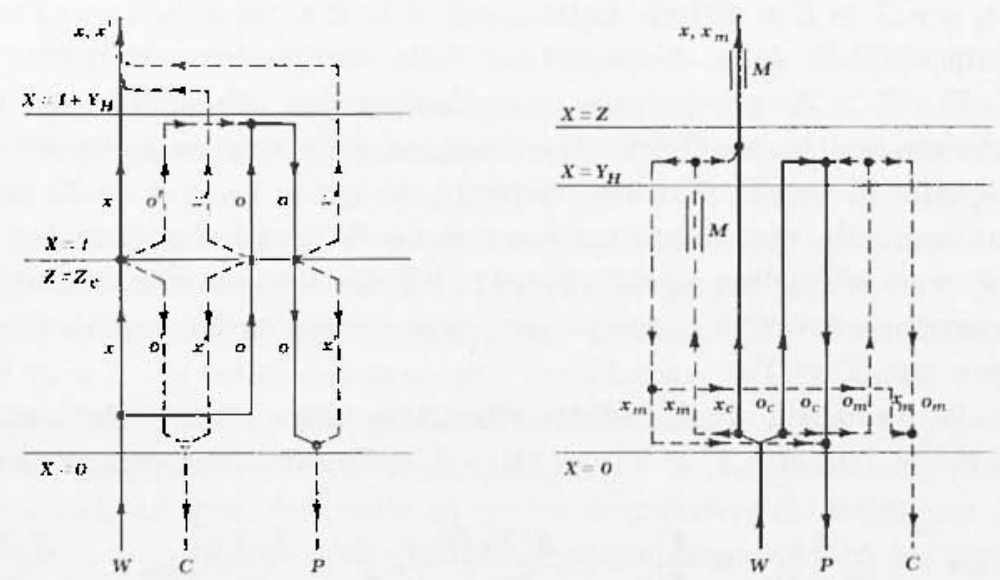

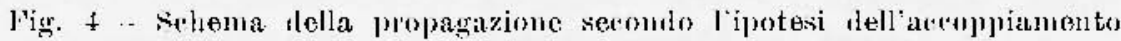
(a sinistro) e vecondo l'ipotexi delle romponenti magnetoinniche modificale (a destra).

alla quota alla quale is $\mathrm{X} \ldots \mathrm{I}+F_{\|}$ha lnogo la riflessione flella componente ordinaria, alla quota alla quale $X=1$ e $Z$ assume il valore (ritico $\left.Z_{c}-Y_{n} \operatorname{sen}^{2} g_{j} / 2 \cos i\right)$ si ecciterebbero componenti ordinaric e straordinarie (classiche) sia progressive sia retrograule, "accoppriate " tra loro in morlo da dar luogo, insieme alle date componenti primarie, a sognali rillessi rome inclionto nella figura (c la la meslesima polarizzazione di If in quanto ad esso contribuiscono componenti della stessa specie, eccitate da quelle di W). Lat situazione in termini rlel precerlente mecranismo ron le componenti modificate è schematizzata nella stessa Fig. 1, a destra (il simbolo $M$ evislenzia la possibilità di intrappolannento nel rono di Storey). Dal punto ali vista delle grandezze sperimentalmente misuabili, è evidente che i flue sclumi sono simili: (io) significa che il modello qui proposto è coerente con quello allaccoppiamento e con i risultati degli esperimenti dianzi ricoralati, ma che al tempo stesso esperimenti di questo tipo non appaiono risolutivi nel far accettare nuo piutitosto che l'altro slei due modelli. it pero 
probabile che una trattazione più estesa ai quella qui appena delineata e suggerita potrà indicare qnilche esperimento cruciale sull'attendibilità delle consegnenze clie abbiamo tratto dalle (48).

\section{BIBLLOGRATIA}

(1) Agnemi G., Chino M., Cutolo M., Pugisi M., "Advan. in Plyys, ", $19,217,1970$.

(2) Dominici P., Radiopropogazione ionosferien, Monografia, Suppl. "Aunali di Geofisica ", XXIV, 1971.

(3) Hediwelt R. A., "Proc. IRE ", 46, 785, 1958.

(4) Helliwell R. A., "Jouru. Geoph. Res, ", 61, 139, 1956.

(б) Parkixison R. W., "Jourm. Atut. Tert. Phys, ", 8, 158, 1956.

(5) Porn J. H., "Jon'n. Gouph. Res. ", 66, 67, 1961.

(7) Ra G., Воокви IF. G., "Journ, Geoph. Res, ", 68, 387, I963.

(अ) Ratctirie J. A., The Wagnetoionic Theory, Cambridge Zniv. Press, 1959.

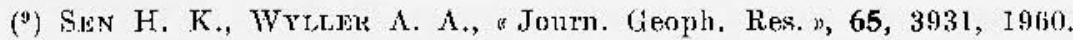

(10) Surtu R. T., IIstamble R. A.. Yabrom I. W., "Journ. Geoph. Res. ", $65,815,1960$.

(11) Stower T. R. O., "Plil. Trans. Roy. Soc. Lomilon" 246, 113, 1953. 